\title{
The Hydrogen Passivated Graphene Cluster and its Stability - First Principle DFT (B3LYP) Levels of Approximation with the Basis Set 3-21G.
}

\author{
Department of Civil Engineering, Kathford International College of Engineering and Management, Lalitpur, Nepal \\ Debendra Baniya \\ Corresponding Email: debendra.baniya@kathford.edu.np
}

\begin{abstract}
First-principles DFT (B3LYP) levels of calculations with the basis set 3-21G have been carried out in order to study the geometric stability and electronic properties of hydrogen passivated graphene (H-graphene) clusters $\left(\mathrm{C}_{\mathrm{N}}\right)$ (where $\mathbf{N}=\mathbf{6}$, $10,13,16,22,24,27,30,35,37,40,42,45,47,48,50,52,54,70$ and 96) and perform the DOS spectrum on $\mathrm{H}$-graphene $\left(\mathrm{C}_{16} \mathrm{H}_{10}, \mathrm{C}_{24} \mathrm{H}_{12}, \mathrm{C}_{30} \mathrm{H}_{14}, \mathrm{C}_{48} \mathrm{H}_{18}, \mathrm{C}_{70} \mathrm{H}_{22}\right.$ and $\left.\mathrm{C}_{96} \mathrm{H}_{24}\right)$ using Mulliken population analysis by the Gaussian $03 \mathrm{~W}$ set of programs. The variations of ground state energy of graphene clusters are observed on sizes and corresponding number of carbon atoms. The binding energy per carbon atom is the function of carbon atoms for the number of carbon atoms less than 30 and saturated at carbon's number 30 and more in the DFT (B3LYP) levels of approximation with the basis set 3-21G. The binding energy per carbon atom of a pure graphene sheet $\mathrm{C}_{32}$ is $8.03 \mathrm{eV} /$ atom in the DFT (B3LYP) level of approximation with the choice of the basis set 3-21G, which is acceptable with previous reported data $7.91 \mathrm{eV} /$ atom. The HOMO-LUMO gap in NBO is studied for some $\mathrm{H}$-grapheneclustors $\mathrm{C}_{16} \mathrm{H}_{10}, \mathrm{C}_{24} \mathrm{H}_{12}$, $\mathrm{C}_{30} \mathrm{H}_{14}, \mathrm{C}_{48} \mathrm{H}_{18}, \mathrm{C}_{70} \mathrm{H}_{22}$ and $\mathrm{C}_{96} \mathrm{H}_{24}$.
\end{abstract}

Keywords-Graphene. H-graphene. Stability. Density Functional Theory (DFT). HOMO-LUMO energy. DOS spectrum. Natural Bond Orbitals (NBOs).

\section{INTRODUCTION}

Carbon atoms appear in many different forms in global environment as a back bone of organic molecules. Both diamond $\left(\mathrm{sp}^{3}\right)$ and graphite $\left(\mathrm{sp}^{2}\right)$ are the pure state of carbon found in nature. They have very different properties, even though they only differ by the arrangement of the carbon atoms. Diamond is insulating and very hard, while graphite is conducting and lubricant [1]. Carbon is crucial to the stability and properties of many natural and artificial structures [2]. In 1985, the first artificial pure carbon material, the spherical molecule called fullerene [3], was found. In 1991, carbon was produced in the form of quasi one-dimensional nano-tubes. Later, in 2004, a flat monolayer sheet of $\mathrm{sp}^{2}$ - bonded carbon atoms tightly packed into a two-dimensional (2D) honeycomb lattice, and is a basic building block for graphitic materials of all otherdimensionalities, has been experimentally discovered, named graphene [4].

Graphene exhibits extraordinary electronic properties and having unique structure its $\pi$ and $\pi^{*}$ bands collapsed together into a single point at the Fermi energy the so-called Dirac point and display a linear E vs. k dispersion. Such an exceptional properties of graphene including high current density, ballistic transport, high thermal conductivity, field emitter, optical transmittance, chemical inertness components of integrated circuits, transparent conducting electrodes, sensors and super hydrophobicity at nanometer scale and the anomalous quantum Hall effect [5], has provoked the scholars for its research field [6]. More, recently, graphene based electronics devices have become realistic, indicative of that the initial thought purposed for graphene can be realized [7]. Graphene has a high mobility charge carriers (electrons or holes) with low electronic noise generated by the thermal agitation of the carriers inside an electrical conductor at equilibrium, which is due to any applied voltage (called Johnson noise), and allowing it in a field effect transistor (FET) as the channel. The high electrical conductivity and optical transparency promote it as transparent conducting electrodes, that required for applications in touch-screens, liquid crystal displays (LCD), organic light-emitting diodes (OLED) and photovoltaic cells. Therefore, it is clear that materials based on graphene will have enormous potentials in electronic devices, surface catalysis, nanotechnology, data storage, and magnetic memory applications [8]-[11].

The first-principles method is used for studying and predicting the properties of atomic and molecular system [12]. They are based upon the fundamental laws of quantum mechanics and use a variety of mathematical transformations and approximation techniques to solve the basic equations [13]. They mainly calculate the ground state energy and the wave functions of the many electron system, which further allows the calculation of various properties like dipole moment, vibrational frequency, optimization energy, nuclear quadrupole moment etc. The first-principles approaches can be classified into three main categories: the Hartree-Fock approach, the density functional approach and the quantum Monte-Carlo approach. One of these approaches, in the present work we have discussed about the density functional theory (DFT), which uses the electron density rather than the wave function for obtaining information about atomic, molecular and solid system [14]. This approach is in principle exact, but the exact form of the exchange correlation potential is not known. In order to perform the $a b$ initio calculations, we have used the Gaussian $03 \mathrm{~W}$ set of programs in lab [15]. In this work, we study for the structural stability of hydrogen passivated graphene clusters (H-graphene) consisting of 6 to 96 carbon atomson the basis set 3-21G by DFT approach. Details of the calculation method Beck-3-parameters-Lee-Yang-Parr hybrid (B3LYP) functional together with the basis set 3-21G [16], [17] are described in Section II. The result of this analysis would help to fit H-L gap on metal-semiconductor properties of graphene. The binding energy (B.E.), binding energy per carbon atom (B.E./atom), density of states (DOS), HOMO-LUMO energy gap are calculated by DFT (B3LYP) approach on the basis set 3-21G in Section III.

\section{COMPUTATIONAL METHOD}

One of the most useful and fundamental approach to take into account of electron correlation is DFT. In DFT, the electronic orbitals are the solutions to a many-electrons Schrodinger equation, which depends upon electron density rather than the one-electron spin orbitals as in the HartreeFock approach. The basic approach of the DFT is to approximate the electron correlation by means of general 
functional of electron density. For this, we first describe the first and second Hohenberg-Kohn theorems [18] and then proceed to discuss the Kohn-Sham approach for the calculation of exchange correlation potential [19].

Now, the electronic Hamiltonian of many-electron system obtained within the Born-Oppenheimer approximation, which can be expressed as

$$
H=T+V_{\text {ne }}+V_{\text {e日 }} \ldots \ldots \ldots \text { (1) }
$$

Where the first term represents the kinetic energy of electrons, the second term represents the Coulomb attraction between electrons and nuclei and the third term is for the Coulomb repulsion among electrons. The second term in (1) for $N$ electrons and $M$ nuclei system can be written as

$$
V_{\text {ne }}=\sum_{\mathrm{i}=1}^{\mathrm{N}} V_{\text {ext }}\left(\mathrm{G}_{\mathrm{i}}\right)
$$

Where, $V_{\text {oxt }}\left(r_{i}\right)=\sum_{i=1}^{W} \frac{z_{n}}{\left|r_{i}-R_{n}\right|} \ldots \ldots$ (3)

is called the external potential on the $i^{\text {th }}$ electron due to $M$ nuclei present in the system. With this Hamiltonian, the ground state energy can be written as

$$
\begin{array}{r}
E_{0}=\left\langle\Psi_{0}|T| \Psi_{0}\right\rangle+\left\langle\Psi_{0}\left|V_{n v}\right| \Psi_{0}\right\rangle+\left\langle\Psi_{0}\left|V_{e e}\right| \Psi_{0}\right\rangle \\
\text { Or, } E_{0}=T+V n e+V e e
\end{array}
$$

Where $\left|\Psi_{0}\right\rangle$ is the ground state wave function for N-electron system.

The DFT calculations are performed to study the ground state geometry, stability of graphene and adsorption of lithium atom on $\mathrm{H}$-graphene, using the first-principles calculation on Gaussian $03 \mathrm{~W}$ set of programs by the B3LYP exchange correlation functional and 3-21G basis set.

The stability of H-graphene with number of carbon atoms is studied on the DFT (B3LYP) levels of approximation. To our best knowledge, a systematic study of the ground state energy of a graphene sheets could not found as a function of number of carbon atoms in using first -principles method. The results obtained for the ground state energy and binding energy per carbon atom on different $\mathrm{H}$-graphene sheets are presented in this study. Moreover, it also discusses the population analysis with the charge distribution Natural Bond Orbitals (NBO), HOMO-LUMO (Highest Occupied Molecular Orbital- Lowest Unoccupied Molecular Orbital) gap. HOMO-LUMO is the energy difference between the highest occupied energy level molecular orbitals and the lowest unoccupied energy level molecular orbitals.

$\mathrm{H}$-graphene cluster was optimized at first without any symmetric restriction for the study of the stability of $\mathrm{H}$ graphene by using Gaussian $03 \mathrm{~W}$ set of programs with the basis set $3-21 \mathrm{G}$. The optimize geometry was used to calculate the frequency for each cluster. With global minima, these frequencies for each cluster were real, ensuring each optimize structure. The binding energy of $\mathrm{H}-$ graphene has been calculated by using the relation;

$$
E_{\text {DindingEnergy }}=E_{H-\text { graphene }}-N_{C} E_{\text {Carbonow. }} \text { (5) }
$$

Where, $N_{C}$ is the number of Carbon atoms in the H-graphene cluster, $E_{\text {Carbon }}$ is the ground state energies of isolated Carbon atom and $E_{H \text {-graphene }}$ is the ground state energy of corresponding $\mathrm{H}$-graphene cluster.
The binding energy per carbon atom has been calculated as the binding energy of $\mathrm{H}$-graphene cluster divided by the total number of carbon atoms $\left(N_{C}\right)$ in the corresponding $\mathrm{H}$ graphene cluster:

$E_{B, E \text {.per carbon atom }}=\frac{E_{\text {BirdinqEneygy }}}{N_{C}} \ldots \ldots$ (5)

\section{RESULTS AND DISCUSSION}

[1] The Binding Energy and Stability of Graphene The optimization of graphene clusters $\left(C_{N}\right)$ is performed with the different numbers of carbon atoms $(N=6,10,13$, $16,19,22,24,27,30,32,35,37,40,42,45,47,50,52,54$, 70 and 96 ) and their corresponding ground state energies are obtained. The geometrical stability (optimization) of $\mathrm{C}_{16} \mathrm{H}_{10}$, $\mathrm{C}_{24} \mathrm{H}_{12}, \mathrm{C}_{30} \mathrm{H}_{14}, \mathrm{C}_{48} \mathrm{H}_{18}, \mathrm{C}_{70} \mathrm{H}_{22}$ and $\mathrm{C}_{96} \mathrm{H}_{24} \mathrm{H}$-graphene is studied in the figure 1 . The variation of the binding energy of $C_{N}$ by using (5) with the number of carbon atoms on the DFT (B3LYP) levels of approximation on the choice of basis set $3-21 \mathrm{G}$ in figure 2 . It shows that the binding energy of graphene cluster increases on increasing the numbers of carbon atom and the size of graphene cluster. On studying the stability of graphene, the binding energy per carbon atom is estimated by using (6). The binding energy per carbon atom of graphene clusters in the DFT (B3LYP) levels of approximation with 3-21G basis set increases with increase in the number of carbon atoms $\left(N_{C}\right)$ on cluster size and tending toward saturation. So, the larger size $\mathrm{H}$ graphene clusters are relatively more stable than smaller sized as shown in figure 3.

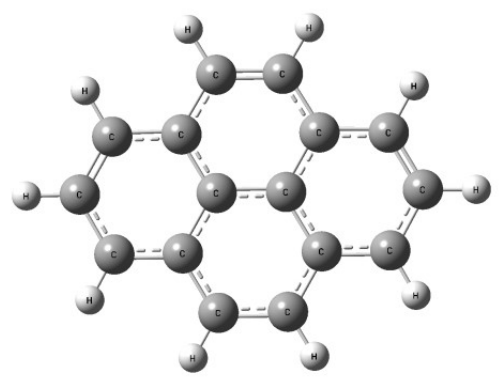

(a) $\mathrm{C}_{16} \mathrm{H}_{10}$

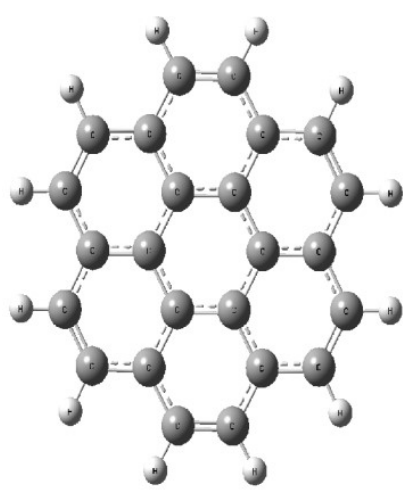

(b) $\mathrm{C}_{24} \mathrm{H}_{12}$ 


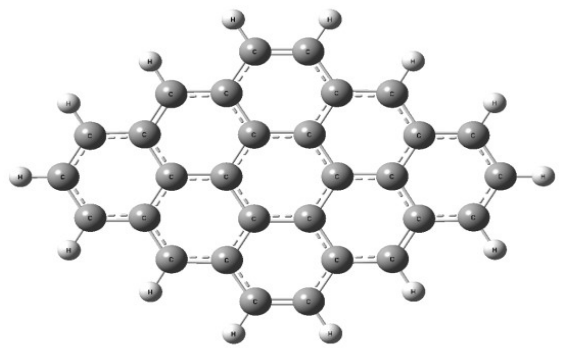

(c) $\mathrm{C}_{30} \mathrm{H}_{14}$

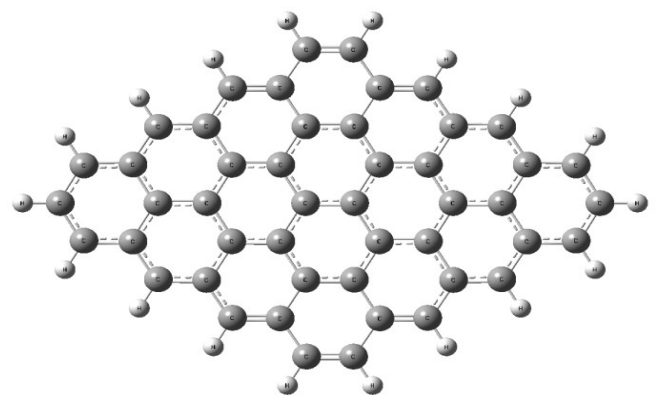

(d) $\mathrm{C}_{48} \mathrm{H}_{18}$

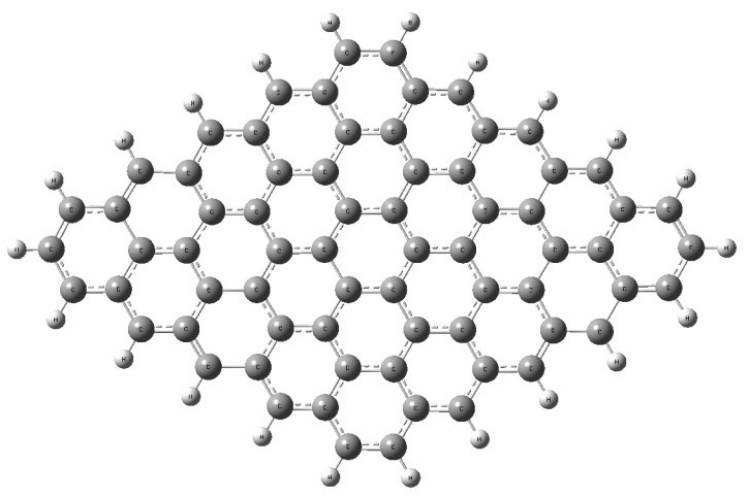

(e) $\mathrm{C}_{70} \mathrm{H}_{22}$

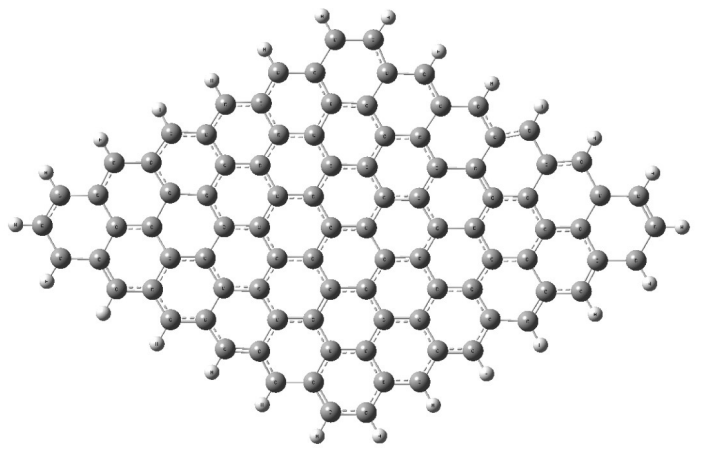

(f) $\mathrm{C}_{96} \mathrm{H}_{24}$

Fig 1.Optimized geometries of H-graphene clusters (a), (b), (c), (d), (e) and (f), in which each carbon atom on the edge of the clusters has been passivated with Hydrogen atom and then were subjected to optimization using DFT(B3LYP) with the basis set 3-21G. For each clusters, the Mulliken charge amount (positive or negative) of each atom increases if that atom is nearer to the edge.
The variation of ground state energy with number of carbon atoms is shown in figure 2, which shows that the ground state energy decreases linearly with the increasing in number of carbon atoms. The best fitted linear equations for the ground state energy of graphene clusters as a function of number of carbon atoms $\left(N_{C}\right)$ is $E\left(C_{N}\right)=0.33753$ $37.87809 N_{C} \mathrm{eV}$ in the DFT (B3LYP) level of approximation with 3-21G basis set. The energy has been measured in units of Hartree.

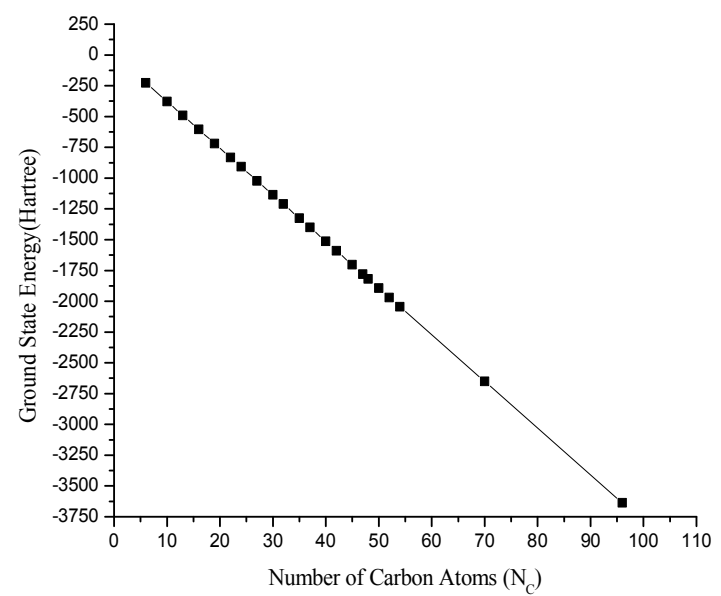

Fig 2.The variation of the ground state energy of the graphene clusters $\left(\mathrm{C}_{\mathrm{N}}\right)$ with the number of carbon atoms $\left(\mathrm{N}_{\mathrm{C}}\right)$ in the DFT (B3LYP) by considering 3-21G level of approximation. The best fitted line is: $E$ $\left(C_{N}\right)=0.33752-37.87809 N_{C} e V$

Figure 3 shows the variation of the binding energy (B.E.) of graphene clusters $\left(C_{N}\right)$ with the number of carbon atoms $\left(N_{C}\right)$ using the DFT (B3LYP) levels of approximation with the basis set 3-21G. The binding energy of graphene clusters increases with increase in their size. The linear equation of the variation of binding energy with number of carbon atoms in DFT approximation is B.E. $\left(C_{N}\right)=-9.22033+$ $8.30515 N_{C} \mathrm{eV}$. By using this equation, we would be estimated the B.E. for any H-graphene cluster with corresponding number of carbon atoms.

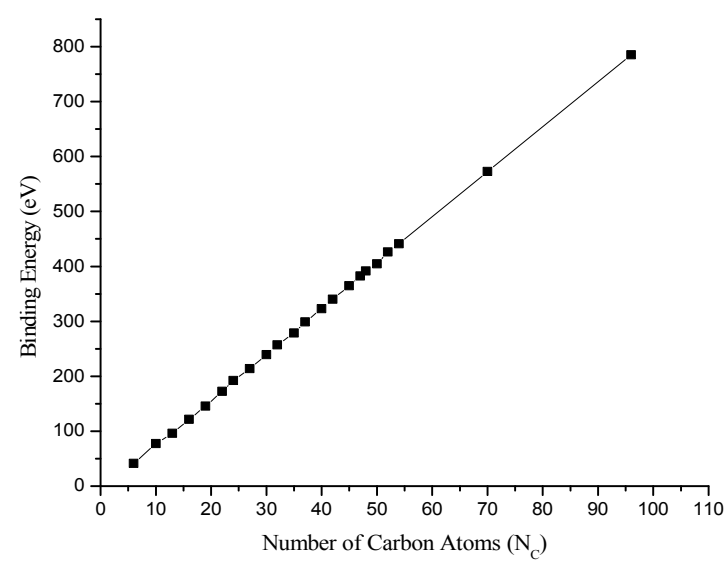

Fig.3. The variation of the binding energy of the graphene clusters $\left(C_{N}\right)$ with the number of Carbon atoms $\left(N_{C}\right)$ using the DFT(B3LYP) levels of approximation with $3-21 \mathrm{G}$ basis set. Best fitted line B.E. $\left(C_{N}\right)$ $=\left(-9.22033+8.30515 N_{C}\right) \mathrm{eV}$ 


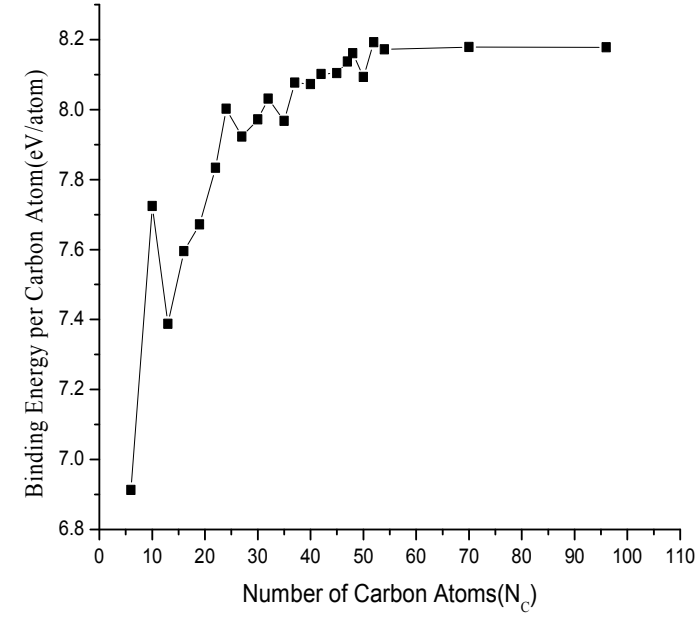

Fig.4. The variation of binding energy per carbon atom (eV/atom) of graphene clusters $\left(\mathrm{C}_{\mathrm{N}}\right)$ with the number of carbon atoms $(\mathrm{N})$ in the DFT (B3LYP)/3-21G level of approximation.

The binding energy per carbon atom of graphene $\mathrm{C}_{10}$ and $\mathrm{C}_{24}$ are more than those of the neighboring graphene clusters (figure 4) $\mathrm{C}_{6}, \mathrm{C}_{13}, \mathrm{C}_{16}$ and $\mathrm{C}_{22}$. This is because of the fact that for $\mathrm{N}=10$ and 24 graphene sheets are in closed form and more symmetric structures are formed than theirs neighboring graphene clusters. For the graphene sheet that contains carbon number atoms 30 and/or more, the change (increasing or decreasing) in the value of binding energy per carbon atom is approximately $1 \%$ in the DFT (B3LYP) in the same basis set $3-21 \mathrm{G}$. So, it is concluded that the monolayer graphene sheet containing 30 and/or more number of carbon atoms is more stable than the lower size graphene clusters. The binding energy per carbon atom of a pure graphene sheet having 32 number of carbon atoms $\left(C_{32}\right)$ is calculated using (6) is $8.03 \mathrm{eV} /$ atom. The binding energy per carbon is in good agreement with those of PBE calculation, $7.91 \mathrm{eV} /$ atom, which is within the $1.52 \%$ of the previously reported value [20].

\section{[2] HOMO-LUMO (H-L) Energy Gap}

HOMO-LUMO gap is the energy difference between the HOMO and LUMO. Molecular energy is very useful for chemist and physicist. The HOMO regards to ionization potential energy (donate an electron) and LUMO as an electron affinity (accept an electron).The strength and stability of molecules can be estimated by the difference in energy between the two frontier orbitals, as well as the colors in solution [21]. The HOMO energy level refers to an organic semiconductor what the valence band maximum is to inorganic semiconductors and quantum dots. The same analogy exists between the LUMO level and the conduction band minimum [22].By the frontier electron density, H-L energy gap is used for estimating the most reactive position in $\pi$-electron systems and explain several types of reaction in conjugated systems too [23].
In this work, the structural dependence of H-L energy gap of some H- graphene clusters is studied by using NBOs analysis. H-L energy gap decreases with increase in number

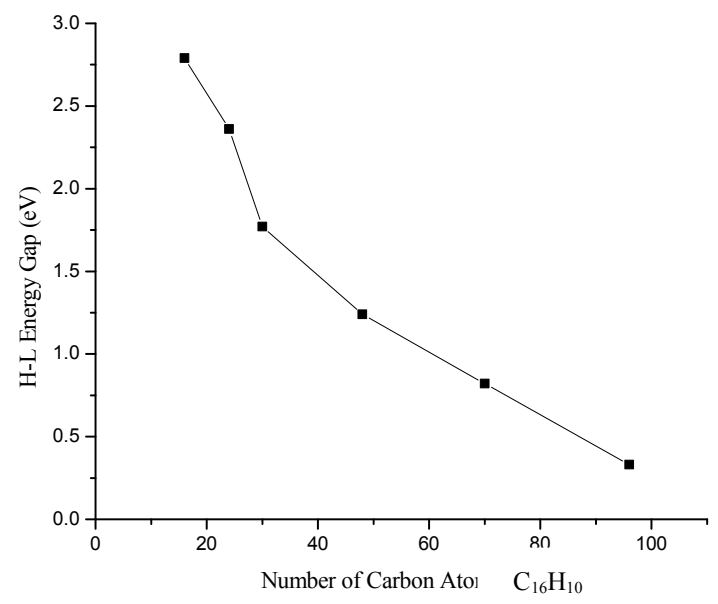

Fig. 5. HOMO-LUMO (H-L) energy gap among H-graphene clusters with number of carbon atoms.

of atom of $\mathrm{H}$ - graphene cluster i.e. H-L energy gap is a negative function of cluster size as shown in figure5. It is acceptable with the previous calculations [24]. From this figure, it is seen that large energy gap is appeared for small size cluster, because of the significance of quantum effect in small size $\mathrm{H}$-graphene clusters. For large size of H-graphene cluster, H-L energy gap is very small and so the quantum effect becomes insignificant. In general, the H-L energy gap would be zero for too large size of $\mathrm{H}$-graphene clusters and it concludes that its electronic properties would be same as that of pure graphene.

The DOS spectrum for each H-graphene cluster is plotted by using Gauss Sum 3.0 software [25] under the basis of Mulliken Population analysis and the occupied and unoccupied molecular orbitals can be seen of corresponding H-graphene clusters in figure 6. From the figure, the electron levels becomes closer and at the same time fermi level energy increases with increase in cluster size.

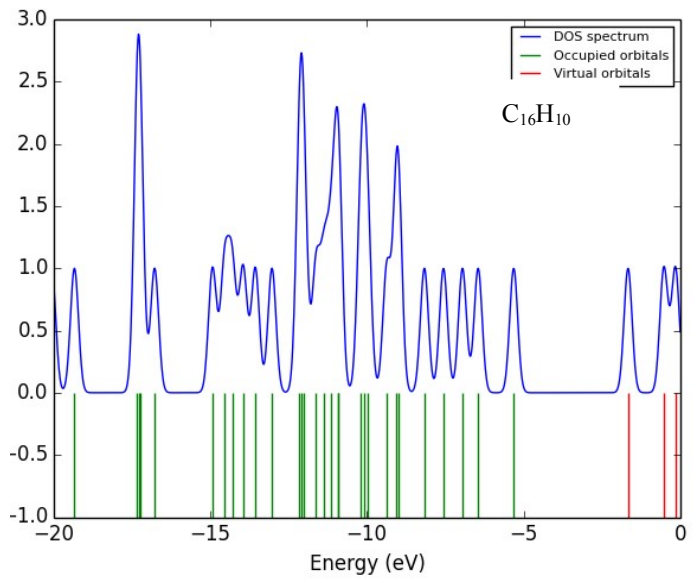



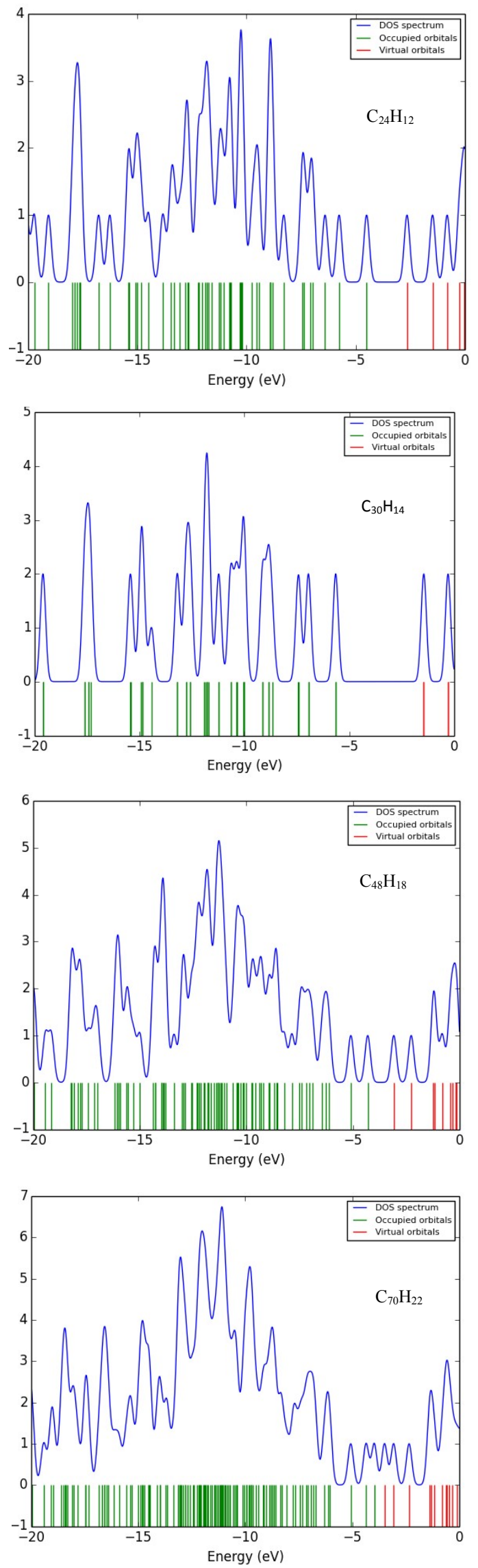

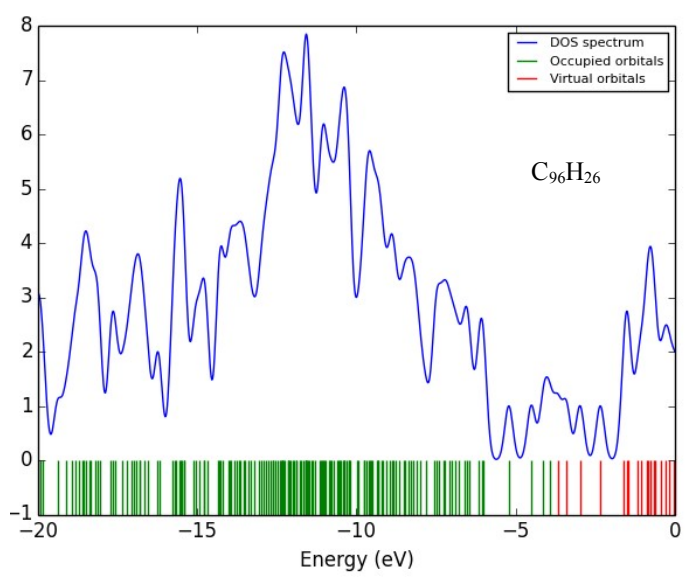

Fig.6. DOS spectrum for $\mathrm{H}$-graphene clusters $\left(\mathrm{C}_{16} \mathrm{H}_{10}, \mathrm{C}_{24} \mathrm{H}_{12}, \mathrm{C}_{30} \mathrm{H}_{14}\right.$ $\mathrm{C}_{48} \mathrm{H}_{18}, \mathrm{C}_{70} \mathrm{H}_{22}$ and $\mathrm{C}_{96} \mathrm{H}_{26}$ ) using Mulliken population analysis with DFT (B3LYP )/ 3-21G levels of calculation.

\section{CONCLUSIONS}

The ground state energy by first-principles DFT (B3LYP) levels of approximation with the choice of basis set $3-21 \mathrm{G}$ is studied and performed structural stability of $\mathrm{H}$-graphene cluster $\left(\mathrm{C}_{\mathrm{N}}\right)$ as a function of corresponding number of carbon atom $\left(N_{C}=6,10,13,16,22,24,27,30,32,35,37\right.$, 40, 42, 45, 48, 50, 52, 54, 70 and 96) using Gaussian $03 \mathrm{~W}$ set of programs. It is found that the ground state energy decreases with increase in number of carbon atoms in graphene sheet in the DFT (B3LYP) levels of approximation with the choice of the basis set 3-21G. Ground state energy is linearly dependent on the number of carbon atoms, so linear equation for the ground state energy of $\mathrm{H}$-graphene as a function of number of carbon atoms $\left(N_{C}\right)$ is $E\left(C_{N}\right)=$ $0.33752-37.87809 N_{C} \mathrm{eV}$ in figure 2 . The binding energy of graphene cluster increases linearly on increasing the size of graphene cluster. The linear equation of the variation of binding energy with number of carbon atoms in DFT approximation is B.E. $\left(C_{N}\right)=-9.22033+8.30515 N_{C} \mathrm{eV}$ in figure 3 and the variation of binding energy per carbon atom (eV/atom) of graphene clusters with the number of carbon atoms in figure 4 shows that the monolayer graphene sheet containing 30 and/or more number of carbon atoms is more stable than the lower size graphene clusters and saturated for relatively larger sized H-graphene cluster.

From above calculations, it is also analyzed the population analysis performed by using the Gaussview $4.1 \mathrm{~W}$ set of programs under the NBOs analysis using DFT (B3LYP) level of approximation with the basis set $3-21 \mathrm{G}$. i.e. the charge distribution and HOMO-LUMO gap for the $\mathrm{C}_{16} \mathrm{H}_{10}$, $\mathrm{C}_{24} \mathrm{H}_{12}, \mathrm{C}_{30} \mathrm{H}_{14}, \mathrm{C}_{48} \mathrm{H}_{18}, \mathrm{C}_{70} \mathrm{H}_{22}$ and $\mathrm{C}_{96} \mathrm{H}_{26}$ system using the DFT (B3LYP) level of approximation with the choice of the basis set $3-21 \mathrm{G}$. The natural bond orbitals (NBO) explain the transfer of charge to the $\mathrm{H}$-graphene sheet, important for the adsorption of metal in H-graphene cluster. The HOMOLUMO gap in NBO study for the $\mathrm{C}_{16} \mathrm{H}_{10}, \mathrm{C}_{24} \mathrm{H}_{12}, \mathrm{C}_{30} \mathrm{H}_{14}$, $\mathrm{C}_{48} \mathrm{H}_{18}, \mathrm{C}_{70} \mathrm{H}_{22}$ and $\mathrm{C}_{96} \mathrm{H}_{24}$ clusters are 2.79, 2.36, 1.77, 1.24, 0.82 and $0.33 \mathrm{eV}$ respectively. Also, the binding energy per carbon atom of a graphene sheet $\mathrm{C}_{32}$ is $8.03 \mathrm{eV} /$ atom in the DFT (B3LYP) level of approximation with the choice of the 
basis set 3-21G (figure 4), which is acceptable with previous reported data $7.91 \mathrm{eV} /$ atom and $\mathrm{C}_{70} \mathrm{H}_{22}$ and/or more carbon atom graphene clusters are more stable. The result of the present work will be estimated to study the stability of graphene clusters $\left(\mathrm{C}_{\mathrm{N}}\right)$ with large number of carbon atom on the choice of different basis sets.

\section{REFERENCES}

[1] M. Kasper, K. Siegmann, K. Sattler, and J. Aerosol, Sci. 1569, pg. 28 (1997).

[2] M. Kasper, K. Sattler, K. Siegmann, U. Matter, H. C. Siegmann, and J. Aerosol, Sci. 217, pg. 30 (1999).

[3] W. Andreoni, The Physics of Fullerene-Based and FullereneRelated Materials (Springer, Berlin). pp. 331-379 (2000).

[4] K. S. Novoselov, A. K. Geim, S. V. Morozov, Y. Jiang, S. V. Dubonos, I. V. Grigorieva, and A. A. Firsov, Science. 306, pp. 666-669 (2004)

[5] Zhang, Y.; Tan, Y.W.; Stormer, H.L.; Kim, P. Experimental observation of the quantum Hall effect and Berry's phase in graphene. Nature.438, pp. 201-204 (2005)

[6] A. K. Geim and K. S. Novoselov, Nat. Mater. 6, pg. 183 (2006)

[7] O. Leenaerts, B. Partoens, and F. M. Peeters, Phys. Rev. B: Condens. Matter.Phys. 77, pp. 125-416 (2008).

[8] A. K. Geim, and K. S. Novoselov, The rise of graphene, Nature Mater. 6, pg. 183-191 (2007)

[9] CastroNeto, A.H.; Guinea, F.; Peres, N.M.R.; Novoselov, K.S.; Geim, A.K. The electronic properties of graphene. Rev. Mod. Phys.81, pg. 109-162(2009).

[10] Seneor, P.; Dlubak, B.; Martin, M.B.; Anane, A.; Jaffres, H.; Fert, A. Spintronics with graphene. MRS Bull.37, pp. 12451254(2012)

[11] Das Sarma, S.; Adam, S.; Hwang, E.H.; Rossi, E. Electronic transport in two-dimensional graphene. Rev. Mod. Phys. 83, pp. 407-470 (2011).
[12] S. M. Blinder, Basic Concepts of self-consistent field theory; American journal of physics, 33(6), pg. 431, (1965).

[13] W. Yang and R. G. parr, Density Functional Theory, Oxford University press, New York; 1989.

[14] Ira N. Levine. Quantum chemistry, Dorling Kindorsley (india)Pvt.Ltd,F.I.E., patparganj, New Delhi110092, India, 5th ed.482(2006).

[15] M. J. Frisch, G. W. Trucks, H. B. Schlegel, G. E. Scuseria, M. A. Robb, J. R. Cheeseman, J. A. Montgomery, Jr., T. Vreven, K. N. Kudin, J. C. Burant, J. M. Millam, S. S. Iyengar, J. Tomasi, V. Barone, B. Mennucci, M. Cossi, G. Scalmani, N. Rega, G. A Petersson, H. Nakatsuji, M. Hada, M. Ehara, K. Toyota, R.Gaussian 03 W, Revision E.01 (2003).

[16] [16] A. D. Becke, Density functional thermochemistry. III. The role of exact exchange, J. Chem. Phys. 98, pg. 5648 (1993).

[17] C. Lee, W. Yang, and R. G. Parr, Development of the ColleSalvetti correlation energy formula into a functional of the electron density, Phys. Rev. B 37, pp. 785-789 (1988).

[18] P. Hohenberg and W. Khon, Inhomogeneous Electron Gas, Physical Review, 138(3B), pg. 864 (1964).

[19] W. Khon and L. J. Sham, Self-Consistent Equation Including Exchange and correlation Effects, Physical Review, 140(4A): pg. A1133 (1965).

[20] A. Bhattacharya, S. Bhattacharya, C. Majumder, G.P. Das, Transition-metal decoration enhanced room-temperature hydrogen storage in a defect-modulated graphene sheet. J. Phys. Chem. C 114, pp. 10297-10301 (2010).

[21] Griffith, J.S. and L.E. Orgel. "Ligand Field Theory". Rev. Chem. Soc. Q. 11, 381-383(1957)

[22] [Bredas, J-L "Mind the gap!". Mater.Horiz. 1, pp. 17-19 (2014)

[23] C. H. Choi, M. Kertez, J. Phys. Chem. A, 101 (1997).

[24] M. Lonfat, B. Marsen, and K. Sattler, Chem. Phys. Lett. 313, pp. 539-543 (1999)

[25] N. M. O'Boyle, A. L. Tenderholt, and K. M. Langner, J. Comp. Chem. 29, pp. 839-845 (2008) 